\title{
Proactive architecture for Internet of Things (IoTs) management in smart homes
}

\begin{abstract}
Smart homes are driven by heterogeneity in nature and consist of diverse components that promote user comfort and security. In recent times, tremendous growth of Internet of Things (IoTs) applications is seen in smart homes. The huge diversity of various IoTs applications generally leads to interoperability requirements that need to be fulfilled. Current IoTs management is achieved using physical platforms that lack intelligence on decision making. A proactive architecture that deploys Event-Condition-Action (ECA) method is proposed to resolve the management of heterogeneous IoTs in smart homes. The proactive architecture, developed with a core repository stores persistent data of IoTs schema, proved to be an ideal solution in solving interoperability in smart homes.
\end{abstract}

Keyword: Smart homes; Internet of Things (IoTs); Proactive architecture 